\title{
Knowmads. Innovaciones y retos para la inclusión social en la educación digital no formal en México
}

\author{
Knowmads. Innovations and challenges for social inclusion in non- \\ formal digital Education in Mexico
}

RECIBIDO 23/9/2020 ACEPTADO 12/10/2020 PUBLICADO 1/6/2021

\section{César Correa Arias}

Departamento de Estudios sobre el Recurso Humano, Universidad de Guadalajara, México cesarca@hotmail.com

\section{Maira Beatriz García Híjar}

Departamento de Intervención Educativa, Universidad Pedagógica Nacional, México

maira.garcia@upn142.edu.mx

\section{RESUMEN}

El presente estudio analiza las relaciones que existen entre las prácticas de los nuevos movimientos educativos mediados por las redes sociales virtuales, los límites y oportunidades de inclusión social en los procesos de formación virtual no formal e informal y las políticas educativas en la educación formal en México.

En general, los movimientos educativos, mediados por la virtualidad, han sido considerados como un sistema paralelo a los programas presenciales tradicionales dentro y fuera de las instituciones de educación superior, sin tomar en cuenta su naturaleza y especificidad.

Basamos nuestro análisis en tres dimensiones: a) socio-tecnológica; b) política; y c) sociocognitiva. Este estudio de caso analiza las comunidades no formales mediadas por las redes sociales virtuales localizadas en la Zona Metropolitana de Guadalajara, Jalisco, México. Por constituirse en una población representativa de la educación no formal, se analiza la asociación educativa, Liderazgo Internacional A.C. (LI). Se aplicaron entrevistas a profundidad semi-estructuradas, grupos focales y se realizó un análisis documental de la asociación.

Los hallazgos indicaron que el movimiento educativo LI (con presencia nacional e internacional) y mediado por redes virtuales, se comporta, de manera similar a otros movimientos de esta naturaleza, como incubadoras de "trabajadores del conocimiento" o knowmads. Estos sujetos utilizan las tecnologías como vehículos para la inclusión social, la movilización de procesos de identidad institucional y personal y el desarrollo profesional. Por lo que es urgente que el sistema de educación superior mexicana estimule, favorezca y optimice procesos educativos y políticas de inclusión social aplicables a todos los ciudadanos, desde diferentes configuraciones educativas de manera constante, sin importar los itinerarios de formación de donde provengan (formales, informales o no formales).

PALABRAS CLAVE alfabetización tecnológica, redes sociales virtuales, políticas educativas, educación a lo largo de la vida, educación no tradicional, inclusión educativa. 


\section{ABSTRACT}

This paper analyzes the relations among the practices of new educational movements mediated by virtual social networks, the limits and opportunities of social inclusion in non-formal and informal education and the educational policies of formal education in Mexico.

Generally, the educative movements mediated through virtual education have been considered as a parallel system to face-to-face traditional programs within higher education institutions without taking into account its nature and specificity.

We based our analysis according to three dimensions: a) a socio-technological dimension; b) a political dimension; and c) a socio-cognitive dimension. This case study focuses the analysis in non-formal educational communities mediated by virtual social networks within the Guadalajara Metropolitan Area, Jalisco, Mexico. As a representative sample of population of a non-formal education, we studied the case of the educational association, Liderazgo Internacional A.C. (LI). The research used semi-structured in-depth interviews, focus groups and documental analysis of the association. The findings indicated that the educational movement LI (with national and international representation), mediated by virtual social networks serve as incubators for "knowledge workers" or knowmads (as similar educational movements do). These subjects use technologies as vehicles for social inclusion, mobilization of institutional and personal identity processes and professional development. Consequently, it is urgent that Mexican higher education system stimulates, favors and optimizes educational processes and social inclusion policies applicable to all citizens from different educational settings on a constant basis, regardless of the training itineraries from which they come (formal, informal or non-formal).

KEYWORDS technological literacy, social media, educational policies, lifelong learning, nontraditional education, equal education.

\section{INTRODUCCIÓN}

\subsection{Educación y sociedades tecnologizadas}

La educación formal pública, estandarizada, sistémica, laica y obligatoria de la gran mayoría del siglo pasado había permanecido con pocas variaciones en el orden tecnológico, reformando apenas algunos procesos, pero conservando, sin alteraciones significativas, su estructura general y elementos característicos. Aunado a esto, la escuela había sido concebida por los ciudadanos como un artefacto de certificación de saberes, que aseguraba la apertura a nuevas posibilidades y oportunidades de empleabilidad legitimadas socialmente.

Sin embargo, resulta obvio que los avances en la educación virtual han generado transformaciones relevantes en el sistema educativo formal, desde la última década del siglo pasado. Este proceso de virtualización educativa, considerado anteriormente como complementario y marginal, ha efectuado un giro forzoso masivo y global más que incentivado, debido a las condiciones sociales y sanitarias impuestas por el fenómeno pandémico del COVID-19 de inicios del año pasado, que ha acelerado un cambio cualitativo en el uso fundamental y obligatorio de las tecnologías en la educación formal. Así, una educación tradicional resulta descontextualizada, poco funcional y ciertamente inoperante, para una sociedad mundial con necesidades diversas globalizadas y localizadas en cuanto a formas de aprender, legitimar y validar conocimientos. Como menciona Pozuelo (2014), "si el contexto y las formas de aprender cambian, resulta lógico pensar que la educación debe experimentar modificaciones" (p.18). 
Es evidente la presencia en todo el mundo, de diversos movimientos educativos, generados por sujetos autónomos, que buscan la liberación de la educación institucionalizada y controlada por el Estado y de la estandarización de los procesos de enseñanza y aprendizaje, ante la poca efectividad de las políticas educativas para avalar conocimientos adquiridos fuera de la escuela. El monopolio de la validación y certificación de una educación superior presencial o virtual, institucionalizada por parte del Estado o de algunas agencias o asociaciones privadas y privilegiadas, se constituye en un factor de exclusión social. Las circunstancias educativas y sociales han cambiado, por tanto, aquello que se legitima al interior de las comunidades de conocimiento formales, informales o no formales, debe ser tomado como factor de transformación de las políticas y prácticas educativas.

Esta investigación presenta elementos que evidencian una necesaria transición inclusiva, dentro de los procesos de enseñanza y de aprendizaje en medios no institucionalizados o no reglados por el Estado o las agencias de educación certificadoras. Aspecto que, si bien no anula del todo, la brecha entre la escuela y la vida, al menos las integran en un horizonte significativo. Lo anterior queda reflejado coloquialmente en la expresión popular: "aprobado en la escuela, reprobado en la vida".

En el caso específico de Guadalajara (México) y su zona metropolitana (ZMG), la ahora llamada "Capital de la Innovación", por el rápido desarrollo de su industria tecnológica, generó cambios vertiginosos en la academia, la investigación, el aprendizaje y el ámbito cultural, resultando en una creciente necesidad de actualización y especialización de competencias y habilidades que sobrepasaron las posibilidades de las instituciones de educación superior (Rodríguez, \& Vicario, 2018). Paralelamente, se conformaron diversos movimientos educativos que operan en México como Hacker's Garage, Social Valley, Fuck Up Nights, Start Up Weekend, Campus Party, Talent Network, Epic Queen, Greenmakers, Hacker Traders, Hackatones y varias decenas más de comunidades con miles de miembros que auspician prácticas educativas informales y no formales mediadas parcial o totalmente por las tecnologías digitales.

La investigación se focaliza en una asociación civil de estudios no formales con sede en Guadalajara y cobertura en Latinoamérica y Estados Unidos: Liderazgo Internacional A.C. (LI), por su representatividad, cobertura y calidad. La función de LI se focaliza en la formación de líderes y conferencistas desde hace más de 20 años.

\section{PROBLEMA DE INVESTIGACIÓN}

Denotamos la exclusión político-administrativa de comunidades educativas en México, por parte de la Secretaría de Educación de México, para acceder al reconocimiento, certificación y oficialización de conocimientos y prácticas educativas desescolarizadas lideradas por comunidades educativas no oficiales, que median sus conocimientos total o parcialmente a través de las redes sociales virtuales.

\subsection{Antecedentes}

La integración del uso de las redes sociales virtuales dentro de los procesos de formación en las escuelas en México y la utilización de dispositivos móviles con gran capacidad de almacenamiento, apuntan a una renovación necesaria en la planeación y recursos didácticos intra/extra áulicos. De allí que se evidencias como la falta de sensibilización y capacitación para la integración del uso de las redes sociales en los pro- 
cesos de formación y la poca legitimación gubernamental de estas redes en todas las modalidades educativas (Basantes et al., 2017). Sumado a esto, a partir de la pandemia causada por el COVID-19, la modalidad presencial, en todos los niveles educativos, se detuvo abruptamente a principios del año 2020 obligando a estudiantes, profesores y administrativos a confinarse en sus casas y continuar de manera virtual con los cursos académicos, sin contar con una orientación y capacitación para todo el personal docente, en una transición a la educación mediada por plataformas educativas virtuales y la consideración de las condiciones de exclusión que este cambio genera.

Dicho suceso evidencia la incapacidad de continuar con un modelo magistral, aun en la virtualidad, dominado por la sincronicidad temporal y espacial y la arbitraria homologación de los intereses y necesidades socio-educativas de los estudiantes. Un modelo virtual/tradicional, de este tenor, limita el ingreso y permanencia a un sistema formativo y no se ajusta a la racionalidad y necesidades particulares de los ciudadanos. Adicionalmente, la apropiación de las tecnologías actuales no significa, dentro de los procesos de formación, que los jóvenes sean per natura, nativos digitales. Es decir, que no nacen alfabetizados digitalmente, como los caracterizaba Prensky (2001), sino que van adquiriendo conocimientos intuitivos en las redes sociales para la socialización y visualización de contenidos, pero, dentro de los procesos formativos, se ven limitados a realizar un proceso de aprendizaje en portales virtuales y a utilizar herramientas didácticas para el aprendizaje en aplicaciones digitales.

Como podemos constatar en plataformas educativas virtuales como OffSchooling.com o Miriadax, entre otras muchas, la educación no formal puede ajustarse a un modelo tecnológico centrado en las necesidades de los estudiantes, de una manera más flexible y más comprensiva que en la educación tradicional. Lo anterior, aunado a que las TIC han potenciado de igual manera el aprendizaje en contextos tradicionales, desafía la noción "de que las instituciones educativas formales son el único contexto en el que se produce el aprendizaje" (Engel et al., 2018, p. 131). En consecuencia, se requiere una alianza entre las tecnologías y los procesos formales e informales de adquisición y movilización de saberes y aprendizajes. En este tenor, han nacido, desde la década pasada, diversos movimientos educativos como "Educación expandida", "Aprendizaje Invisible”, “Edupunk” y “Edupop”, etc., que superan las restricciones de las instituciones tradicionales, al fusionar herramientas de investigación e innovación social y educativa mediados por la virtualidad, mediante proyectos multidireccionales y prácticas de aprendizaje informal multicontextuales (CCCB- Centro de Cultura Contemporánea de Barcelona, 2010).

Estos movimientos educativos basan sus prácticas en una mezcla de matices constructivistas (Coll, 2001; Piaget, 1970; Vigotsky, 1978), conectivistas (Siemens, 2005) y cognitivistas (Halpern, 2003; Krathwohl, 2002) para potenciar el aprendizaje inclusivo ciudadano. En este sentido, Correa (2010) afirma que:

Uno de los elementos estratégicos que nos brindan las redes sociales, con respecto a la enseñanza y el aprendizaje es la posibilidad de interactuar con otras culturas y ser incluidos en los distintos espacios sociales, sin necesariamente contar con el amparo de instituciones tradicionales oficiales cuyas acciones limitan las relaciones basadas en procesos jerárquicos y, donde muchas de estas relaciones, no están definidas por la tecnología, sino por la calidad de las prácticas sociales y educativas de profesores, estudiantes y administrativos. (p. 2)

Estas formas educativas modernas influencian la academia de manera relevante. Litto (2012) afirma que la mayor parte del aprendizaje sucederá cuando la necesidad por la información surja, donde las orga- 
nizaciones y movimientos con estructuras ágiles acapararán gran parte del mercado educativo y los cursos formales de la educación a distancia actual y, por tanto, co-existirán con actividades de aprendizaje sin estructuras escolarizadas.

En el contexto mexicano, estas prácticas de enseñanza aprendizaje vienen influenciando las políticas educativas a partir del año 2000, en tanto que la Secretaría de Educación Pública empieza a reconocer, validar y legitimar el aprendizaje autodidacta.

El Acuerdo 286 de la Secretaría de Educación Pública (SEP) de México, incluye algunas políticas que permiten la acreditación de conocimientos adquiridos en forma autodidacta que se corresponden con niveles educativos o grados escolares específicos, a través de la experiencia laboral o con base en el régimen de certificación referido a la formación para el trabajo (SEP, 2000, p. 13).

Lo anterior no garantiza que la educación informal y no formal se convierta en un adalid de inclusión de los sujetos a los mercados de trabajo o que se vincule, de manera más efectiva, que la educación formal, a las necesidades particulares de los estudiantes de manera transversal, en el sistema educativo mexicano.

\section{OBJETIVO DE LA INVESTIGACIÓN}

Analizar el papel de los procesos de formación no formal e informal mediados por ambientes virtuales, en la readecuación de las políticas educativas en México y la calidad de las prácticas educativas de Liderazgo Internacional, A.C, a fin de promover el reconocimiento y valoración de nuevos movimientos educativos, como espacios legítimos de generación y movilización de experiencias educativas efectivas.

\section{FUNDAMENTACIÓN TEÓRICA}

La inclusión digital sugiere la familiarización y apropiación de las nuevas tecnologías de la información y la comunicación con la intención de aminorar la brecha social y educativa (Organización de las Naciones Unidas para la Educación, la Ciencia y la Cultura, UNESCO, 2017). Al analizar las prácticas de los movimientos educativos actuales, desde una dimensión socio-tecnológica, se evidencia la potencialidad de una educación autónoma y flexible para facilitar el desarrollo de las habilidades sustanciales del ciudadano. De allí, el surgimiento de la categoría social de "trabajador del conocimiento" o knowmad (Moravec, 2011).

A este respecto, es relevante el aporte de Moravec (2011), en la teorización de las Sociedades punto cero, al ejemplificar el devenir social mediado por las Tecnologías de la Información y la Comunicación (TIC), desde tres momentos históricos: sociedad 1.0 simboliza a la sociedad industrial, representada por las primeras máquinas de producción en masa. Este tipo de sociedad inflexible, jerárquica y estática influencia en gran medida, los procesos educativos, políticos, laborales y de negocios. De allí que el modelo de la escuela laica, obligatoria y gratuita resultara apropiado, para capacitar obreros trabajadores de las fábricas de la época.

La sociedad 2.0 denominada por Moravec (2011) como: "sociedad del conocimiento", evidencia una colectividad democrática, interaccional, innovativa, heterárquica y cooperativa, debido a la influencia que ejercen las nuevas tecnologías en el orden social actual. Esto es parcialmente cierto pues, aunque se han 
ganado importantes derechos en el terreno social y político, gracias a la aplicación de las tecnologías y que el "aumento, en la adquisición y aplicación de las TIC se ha reflejado significativamente en las actividades cotidianas" (UNESCO, 2017, p. 282), las tecnologías apenas empiezan a responder parcialmente a las necesidades socio-educativas de una ciudadanía global.

A diferencia de algunos clásicos de la sociedad del conocimiento como Boisier (2016), Castells (1995) y Drucker (1994), cuyos fundamentos se basan en la manera cómo el sistema económico imperante condiciona las prácticas sociales, mientras que Moravec (2011) vincula las tecnologías como un factor esencial de las transformaciones cognitivas y sociales actuales.

En esta sociedad los individuos construyen y proyectan al interior de comunidades y movimientos educativos mediados por las redes sociales virtuales, un devenir re-institucionalizado no oficial de prácticas, que son capaces de influenciar profundamente a las instituciones educativas tradicionales.

Así, la importancia política de la adquisición de competencias digitales en comunidades de aprendizaje virtual es evidente, especialmente, porque representa poder, seguridad y apertura. Carretero et al. (2017), las resumen en estas cinco capacidades:

1. Alfabetización en información y manejo de Big data, que articula las necesidades de información para localizar, recuperar y difundir datos, información y contenidos.

2. Comunicación y colaboración para facilitar la interacción, comunicación y colaboración entre comunidades virtuales, respetando la diversidad cultural y generacional.

3. Creación de contenidos digitales, mejorando e integrando información nueva, respetando los derechos de autor.

4. Seguridad, para proteger dispositivos, contenidos, datos personales y la privacidad en entornos digitales.

5. Resolución de problemas, para identificar necesidades y alternativas de solución desde entornos digitales.

Por una parte, los nativos digitales contarían, al menos, con tres o cuatro de estas capacidades. Sin embargo, el desarrollo de las mismas depende, según Linne (2014), de su nivel socio-económico, su capital cultural y la amplitud o limitantes de su entorno tecnológico y se manifiesta en diversos grados de alfabetización tecnológica. De otra parte, los inmigrantes digitales, aquellos que nacieron antes de la era del Internet y que se vieron en la necesidad de aprender a usar las TIC, para insertarse en los diversos ámbitos sociales, se apoyan en:

Sus experiencias cotidianas [...] resuelven un problema a la vez, actúan basados en el análisis deductivo, y el saber está asentado en el conocimiento adquirido previamente. (Jara, \& Prieto, 2018, p. 96)

La sociedad 3.0 (Moravec, 2011), se visualiza como una era de profundas transformaciones en donde se rompe con las estructuras sociales más arraigadas, a causa de un acelerado cambio tecnológico que presenta, entre otras cosas, la globalización constante del conocimiento y su redistribución horizontal. Es decir, donde los contextos tradicionales de profesor-estudiante sufren transformaciones importantes, debido a la liberación de la información disponible para cualquier sujeto y la capacidad de autogestión, del mismo, en un "aprender a aprender" potenciado por los "trabajadores del conocimiento" o knowmads. 
Este término resulta ser una extensión de knowledge worker, el cual surge por primera vez con Drucker (1959), para definir ciudadanos innovadores, creativos, autónomos y sumamente adaptables que integran sociedades ambiguas y caóticas. Estos no dependen de las instituciones o empresas tradicionales, puesto que generan valores agregados y pueden salir y entrar de las mismas al desenvolverse en diversos proyectos, mientras su conocimiento personal y sus redes de conocimiento así se los permita. Son tecnológicamente capacitados para utilizar la información abierta y libremente, crean redes de conocimiento horizontal entre personas y organizaciones, no temen al fracaso y desarrollan un aprendizaje permanente y para toda la vida (formal-informal) (Moravec, 2011).

Estos sujetos difieren de los trabajadores comunes, de la sociedad 2.0, por la movilidad laboral y social que detentan. No requieren de un trabajo estable y la dinámica de transformación e innovación son factores nucleares de su quehacer. De allí que la multitud de oportunidades de trabajo dependan en gran parte de la relación entre desarrollo tecnológico, capacidades para la construcción de redes de conocimiento y el dinamismo de los mercados de trabajo.

Según Gil (2014), el problema del desempleo de profesionistas es consecuencia del modelo de desarrollo de México, como país maquilador más que como generador de conocimiento innovador. La desconexión del ambiente escolar con el mundo laboral se evidencia en una alta deserción escolar en México (50\%, una de las mayores en América Latina), puesto que resulta relativamente fácil emplearse en oficios que requieren mano de obra no capacitada, en contraparte de aquellos que poseen una licenciatura y/o posgrado, cuyo ingreso a los mercados laborales resulta altamente competitivo y restrictivo.

La Organización para la Cooperación y el Desarrollo Económicos (OCDE, 2015), señala que la principal causa de deserción escolar entre los mexicanos es "la falta de interés en la escuela, ya que les servía de poco o era inadecuada para sus intereses y necesidades, argumento seguido de la falta de recursos financieros" (p. 7). Mientras que, en México es posible validar competencias adquiridas en la vida diaria, el número de participantes en los procesos de certificación permanece bajo, al igual que en todos los países miembros de la OCDE (2010).

Una causa aparente de desmotivación es que "la inversión inicial se pierde en caso de no aprobar o desertar antes de la terminación del proceso, aun cuando las razones sean válidas” (OCDE, 2010, p. 58). Por ejemplo, ciertas certificaciones de bachillerato y licenciatura se encuentran entre los MXN 2.000 (100 EUR aproximadamente) y MXN 34.000 (1.700 EUR, aproximadamente), dependiendo de la competencia o grado académico a validar y la fase del periodo de trámites en que se encuentren. Esto es significativo en un país donde el salario mínimo es de MXN 123 (4.71 EUR, aproximadamente) por una jornada de ocho horas.

"El déficit en el capital humano calificado moderno [...] es una de las justificaciones más frecuentes para integrar sistemas que reconozcan los resultados del aprendizaje no formal e informal" (OCDE, 2010, p. 5), de allí, la importancia del uso de plataformas y recursos virtuales como generadores de nuevos espacios de conocimiento e inclusión social. La mayoría de las comunidades mediadas por redes sociales, como LI, son gratuitas o presentan costos simbólicos para cubrir los gastos mínimos de sus operaciones.

Por su parte, Liderazgo Internacional, la asociación analizada, suma en este momento, en Estados Unidos y América Latina, más de 282 clubes y 7800 socios. Entre los socios pasados y actuales han participado senadores, gobernadores y otros miembros en cargos públicos.

Cada club de la asociación, en su respectivo país, designa un día a la semana para realizar una sesión virtual o presencialmente, se fijan un nombre, eligen a un presidente del club y utilizan manuales, creados por la misma asociación. 
Actualmente, tienen una alianza con el Instituto de Estudios Tecnológicos y Superiores Matatipac, A.C. y el Consejo Iberoamericano en Honor a la Calidad Educativa, además, otorgan un "Certificado de Excelencia Académica" con valor curricular, avalado por más de 33 universidades a nivel nacional e internacional para los que concluyen su programa formativo y de prácticas. Dada la emergencia sanitaria actual, esta comunidad mediada por las redes sociales virtuales continúa sus prácticas virtuales únicamente, sin embargo, normalmente proyecta también su ideología y cultura organizacional en eventos sociales, proyectos, congresos y concursos presenciales.

\section{METODOLOGÍA}

El estudio se posiciona en un paradigma formativo-digital, dentro de la tradición hermenéutica. Desde una metodología cualitativa, se aborda un estudio de caso representativo dentro de los movimientos educativos mediados por las redes sociales de la Zona Metropolitana de Guadalajara (ZMG), a fin de analizar cómo esta comunidad comprende y asume sus discursos y prácticas educativas más representativas, mediante la observación directa y la entrevista a los sujetos involucrados (Escudero et al., 2008; Marton, 1992).

Los criterios de selección de los sujetos fueron los siguientes: 1 ) diversidad en los roles de los miembros de la asociación; 2) diferencias demográficas de los miembros; y 3) una antigüedad de los miembros mayor a un año en las actividades de la asociación, con pausas no mayores a un mes.

En la investigación se aplicaron seis entrevistas semi-estructuradas a sujetos claves de la asociación (entre ellos el fundador de la asociación y presidentes de los clubes), dos grupos focales, un registro etnográfico de la interacción de los sujetos con los artefactos físicos, tecnológicos y culturales observados en sus instalaciones y en sus redes sociales virtuales, y un análisis documental de la asociación. Las guías de las entrevistas abarcaron las tres dimensiones centrales de esta investigación: la dimensión socio-tecnológica, política y sociocognitiva.

Respecto a los grupos focales, se realizaron tres sesiones con 9 a 15 individuos, cada uno dentro de las instalaciones de la asociación, en diferentes momentos del trabajo de campo, en días y horarios distintos, para asegurar los mínimos de homogeneidad y heterogeneidad.

\section{RESULTADOS Y DISCUSIÓN}

La investigación reveló que las prácticas educativas en LI se basan en dinámicas de oratoria, donde utilizan la improvisación, la preparación de un discurso y la coevaluación de los demás socios. La totalidad de los miembros pueden acceder a ser parte de los equipos directivos de la asociación. Esta asociación nació con el objetivo de subsanar los problemas de comunicación política y organizacional de los miembros que la integran.

En la sistematización de las transcripciones de las entrevistas y de los grupos focales se identificaron un total de 246 citas, que inicialmente se agruparon en 9 códigos; después, se integraron los códigos de menor densidad (dentro del paréntesis) a esos de mayor densidad (fuera del paréntesis) quedando de la siguiente manera: identidad (trayectoria profesional), cultura (permanencia), itinerarios de formación (certificación y habilidades socio-cognitivas), políticas educativas y legitimación. Así surgieron las categorías de análisis con su definición etic y una clarificación emic para asegurar su confiabilidad y validez. 
Finalmente, se realizó una cartografía y análisis categorial (Correa, 2011a; 2017) para la interpretación de los datos, entre estas cinco categorías de análisis y las cinco categorías conceptuales desarrolladas en la teorización del estudio: sociedades punto cero, tecnología social, reconfiguración de los procesos educativos, alfabetización digital y movimientos educativos.

La totalidad de la investigación incluyó el cruzamiento entre cinco categorías conceptuales y cinco categorías de análisis. Sin embargo, en este presente trabajo, se estudiará la imbricación entre el grupo de categorías: a) Cultura, identidad y tecnología social; y b) Movimientos educativos, reconfiguración de los procesos educativos y alfabetización digital.

\subsection{Cultura, identidad y tecnología social}

La asociación LI, en el marco de una educación no formal mediada por las redes sociales virtuales, promueve una cultura de autogestión, mediante la intervención de los socios en la estructura administrativa.

Las personas que acudimos intercambiamos los puestos de las mesas directivas, se nombran presidentes por cierto periodo, de manera tal que actuemos según nuestros propios criterios, claro, dentro del marco preestablecido. (P5, 2018)

El acceso libre a la tecnología social cambia los roles tradicionales de docentes y alumnos, lo que genera procesos naturalmente distintos, democráticos y heterárquicos que les permite ser una alternativa a los programas de estudio tradicionales (Downes, 2005; Freire, 2010) orientados a la educación a lo largo de la vida, la gestión del conocimiento, la participación cívica y la recreación. Los participantes manejan su ritmo de progreso y realizan actividades co-evaluativas de retroalimentación.

Aquí no hay, por decir, un maestro, todos somos maestros, todos nos ayudamos unos a otros, el personaje más importante se llama socio, y le sigue el club en ese valor, así todos aprendemos de manera colaborativa. (P6, 2019)

En contextos sensibles a las tecnologías emergentes "la formación no reglada, no formal, es cada vez más accesible a través de la red" (García, 2019, p.14).

Es común que en Liderazgo de pronto alguien diga "vi este video, se los recomiendo, es de gran aprendizaje", "te recomiendo este libro que está en Youtube, para que lo lean, habla de eso, podemos aprender de esto", en fin. No cabe duda que el usar la tecnología de manera adecuada, es conocimiento, es apertura, es investigación, es crecimiento, hoy en día, la tecnología es parte fundamental del aprendizaje. (GF, 09)

Defienden la integración de prácticas mixtas similares a los procesos de una educación expandida, donde regla el "abandono de enfoques pedagógicos tradicionales y pasivos [...] con un énfasis en metodologías activas, participativas y colaborativas” (Uribe, 2017, p. 299).

Hay muchas personas que confunden estar en el Internet con estar en Facebook todo el día, platicando todo el rato y no saben que algunas personas sí lo hacen, pero otras se informan, utilizan la Internet para mejorar en su conocimiento y no en su entretenimiento. (GF, 2019) 
En cuanto a su identidad, reportaron recurrir a estos espacios en búsqueda de una mayor autonomía en sus procesos de aprendizaje y calidad de vida, pues reconocen que el sistema educativo, como tal, puede limitar la curiosidad e innovación del individuo. En todo caso, la virtualidad muta el papel y estilo de los enseres pedagógicos, concretamente en la producción de la identidad y el proceso de enseñanza-aprendizaje, pues cohabita con interpretaciones, actuaciones y comunicaciones que revolucionan la percepción tiempoespacio y la identidad del partícipe (Prata et al., 2020):

Liderazgo Internacional, A.C. ha sido para mí el medio perfecto para crecer como persona. Me ha permitido desarrollar y pulir cada una de mis cualidades individuales. A través de las diferentes sesiones semanales presenciales o virtuales, he podido ejercitar mi capacidad de comunicarme eficientemente, mi capacidad de influencia, el sentido de responsabilidad y de compromiso con el cambio personal y de otras personas, que, igual que yo pueden encontrar en la asociación, como un recurso excepcional para la vida. (P3, 2018)

\subsection{Movimientos educativos, reconfiguración de los procesos educativos y alfabetización tecnológica}

Según Potter (2010), Scholari (2016) y la UNESCO (2011), entre otros, la alfabetización tecnológica no es en sí el manejo instrumental de las tecnologías, sino que va mucho más allá: es una práctica sobre todo política, pues comprende aquellos sujetos que utilizan las TIC como una extensión de ellos mismos en sus dominios emocionales cognitivos, estéticos y éticos mediante la cultura y la educación.

\footnotetext{
Yo inicialmente venía a aprender a expresar mis ideas de manera asertiva, sin embargo, al relacionarme con la asociación, especialmente, a través de su página web y de los intercambios presenciales o virtuales, se generó una revolución intelectual, o sea en el nivel neurológico tal vez, no sé, algo pasó porque, también me ha cambiado en mis actitudes y aptitudes. (P7, 2019)
}

Esto sugiere la creación e interpretación de contenidos virtuales de forma crítica, en contraposición a una enajenación digital contraria al knowmad (Scholari, 2016), quien ha adquirido habilidades de investigación, expresión y difusión de información, protección de datos personales y estrategias colaborativas de resolución de problemas en movimientos educativos, mediados por las redes sociales.

Se me hace algo fantástico hoy en día tanta tecnología que tenemos, tanto acceso a la información, al internet y demás, porque desde chicos nos criaron o nos educaron de una manera pasiva, no teníamos tanta motivación para aprender. Las televisoras influyeron mucho en mantenernos estáticos y pasivos, sin interactuar para aprender sino de recibir y recibir contenidos. (P5, 2018)

El aprendizaje móvil o m-learning es una mediación de aprendizaje importante en LI. "La capacidad para ampliar las experiencias educativas más allá de las aulas y permitir el aprendizaje no formal e informal es un atributo clave del aprendizaje por telefonía móvil” (UNESCO, 2015, p. 246).

Yo aprendo mejor fuera de un aula, es difícil concentrarte en un lugar en el que vas a estar dos horas sentado viendo la misma pared. El hecho de no estar interactuando con lo que estás aprendiendo, siento que para mí es una de las limitantes. Ya no es necesario estar sentado en un lugar porque ahí tienes el equipo, las nuevas tecnologías nos dan incluso la posibilidad de tener una clase por fuera de la escuela, sin ningún problema, a través de cualquier dispositivo. (P2, 2018) 
Estas reconfiguraciones de los procesos educativos, presentes en los movimientos efervescentes mediados por las redes sociales virtuales de la ZMG, y específicamente en las prácticas de LI, nos remiten a las teorías iniciales de la desescolarización de Illich (2012), que retratan a la educación efectiva como un proceso que pone a disposición de los interesados, los recursos de aprendizaje en cualquier momento y lugar. Conecta al que desea aprender con el que quiere compartir su conocimiento y ofrece un espacio donde presentar y argumentar un tema generador de debate. Para estos procesos, los miembros de LI utilizan más las redes sociales Facebook, YouTube, Zoom y la página web oficial de la asociación; y mucho menos Instagram, Linkedin y Twitter.

Se infiere entonces que la creación de knowmads depende conjuntamente de la reconfiguración de los procesos educativos y la alfabetización tecnológica, tomando en cuenta que "conceptos como pedagogía, educación, aprendizaje, enseñanza y escuela, entre muchos otros, se están reinterpretando a la luz de las complejas y cambiantes mediaciones que ofrecen las tecnologías modernas” (Uribe, 2017, p. 307).

No sé si a ustedes les ha pasado lo mismo, te sientes completamente más despierto, no únicamente para contestar una pregunta, sino que te haces más consciente de lo que estás hablando, porque el aprendizaje no se queda en la sesión de clase, sino en tu dispositivo, en la internet y te hace consciente de lo que estás diciendo, y lo que llevas a tu cabeza. Despiertas conexiones en tu cerebro que me imagino, estaban dormidas. (GF, 2019)

Finalmente, ningún socio reportó tener conocimiento de la existencia de procesos de certificación de competencias, ni estar interesados. Esto concuerda con las recomendaciones de la OCDE (2010) para implementar mejoras en el sistema de registro en línea y su respectiva promoción. Lo que los atrae a este tipo de opciones educativas son las prácticas autónomas, soberanas, cooperativas y colaborativas que experimentan, y conectan después, con su vida personal. Sin embargo, el contar, además, con una certificación accesible y asequible podría aumentar el interés de la ciudadanía por integrarse a estas comunidades y trabajar en las habilidades del siglo XXI.

\section{CONCLUSIONES}

La pérdida de la escuela como lugar común físico donde ocurren todos los procesos de enseñanza y aprendizaje está obligando a las instituciones a diseñar y optimizar métodos y tecnologías, que permitan la integración de todos los ciudadanos de manera constante y sin prerrequisitos. En virtud de que estas tecnologías promueven la adquisición de competencias y capacidades que están siendo evaluadas de manera más frecuente, por las mismas tecnologías que las proveen, además de generar experiencias heterogéneas, según los fundamentos pedagógicos y didácticos utilizados en el proceso.

Gracias a las prácticas autónomas, autogestivas y multidireccionales, que comportan en los nodos de información y redes de conocimiento, el factor de cambio actual requiere la intervención y colaboración de las comunidades desescolarizadas, mediadas por las redes sociales virtuales y su necesaria conquista de legitimidad. Es importante que se adecuen las políticas educativas en México, a fin de permitir que se validen habilidades, destrezas, y en general, cualquier tipo de conocimiento obtenido en ambientes informales, para construir un modelo inclusivo de empleabilidad y de desarrollo profesional. Esto significa ambientes de aprendizaje donde la presencia de la institución no es tan clara, o donde opera una cierta re-institucionalización de la educación que comprende nuevos territorios del conocimiento (Correa, 2011b, p. 359). 
A pesar de que la educación para adultos puede dotar a los mexicanos de competencias y capacidades relevantes para su vida en sociedad y el mercado de trabajo nacional y mundial, el 75\% reporta poca o nula participación en entrenamientos laborales formales y no formales, ya que los perciben como poco relevantes, laboriosos o costosos (OCDE, 2020). La oferta de opciones accesibles y flexibles centradas en sus necesidades y contextos y, apoyadas por las tecnologías, como la LI, aquí analizada, ejemplifica lo que la cultura digital y autonomía de movimientos sociales puede aportar como apoyo a los gobiernos para adaptarse a la nueva normalidad, no solo ajustada a situaciones coyunturales, sino a espacios de legitimación e inclusión continuos.

No se trata de minimizar los esfuerzos ya realizados en materia política, sino más bien de ofrecer una solución viable a la escasez de espacios de formación, en contraposición a la alta demanda de competencias laborales, los costes que estos generan y su contiguo desinterés social. Las prácticas de movimientos educativos como LI bien pudieran fungir con ese doble rol de instituciones libres (de la pesada solemnidad institucional) capacitadoras y puentes para la inclusión social mediadas por redes virtuales y presenciales con reconocimiento oficial por parte del sistema educativo mexicano.

En el tenor de una trasformación social liderada por trabajadores del conocimiento, los movimientos educativos como LI demostraron ser potenciales incubadoras de knowmads que, a la luz de las tendencias sociales actuales son y serán, los protagonistas de la innovación en todas las esferas de la sociedad 2.0 y 3.0. Lo anterior, señala la urgencia de futuras investigaciones en el campo del aprendizaje desescolarizado mediado por las tecnologías, que abonen al concepto de "educación a lo largo de la vida" como el vehículo de una vida digna para todos y una inclusión socio-educativa relevante y verdadera.

\section{REFERENCIAS}

Basantes, A., Naranjo, M., Gallegos M., \& Benitez N. (2017). Los Dispositivos Móviles en el Proceso de Aprendizaje de la Facultad de Educación, Ciencia y Tecnología de la Universidad Técnica del Norte de Ecuador. Formación Universitaria, 10(2), 79-88. https://dx.doi.org/10.4067/S0718-50062017000200009

Boisier, S. (2016). Sociedad del conocimiento, conocimiento social y gestión territorial. Interações (Campo Grande), 2(3), 9-28. http://dx.doi.org/10.20435/interações.v2i3.583

Carretero, S., Vuorikari, R., \& Punie, Y. (2017). DigComp 2.1: The Digital Competence Framework for Citizens with eight proficiency levels and examples of use. https://publications. jrc.ec.europa.eu/repository/bitstream/JRC106281/webdigcomp2.1pdf (online).pdf

Castells, M. (1995). La ciudad informacional: tecnologías de la información, reestructuración económica y el proceso urbanoregional. Alianza.

Centre de Cultura Contemporània de Barcelona (CCCB) (2010, Febrero 2). Educación expandida |I+C+i. [Web log post]. e http:// www.cccb.org/rcs gene/FULL DE MA ICI EDUEX Esp.pdf
Coll, C. (2001). Constructivismo y educación: la concepción constructivista de la enseñanza y el aprendizaje. En C. Coll, J. Palacios \& A. Marchesi (comps.), Desarrollo psicológico y educación 2. Psicología de la educación escolar, (pp. 157-186). Alianza.

Correa, C. (2010), Collaborative Academic Work as a Power Strategy for an Inclusive E-learning Education. In ICEMT (Ed.), Proceeding in 2010 International Conference on Education and Management Technology. (pp. 62-66)). Press Singapore.

Correa, C. (2011a), Public Policies on Higher Education: Rhetoric and Grammar of Modernity and the limits of social recognition. The International Journal of Interdisciplinary Social Sciences. 5(9), 177-190.

Correa, C. (2011b). Technologies and plural Citizenship. Social recognition within virtual educational communities in Higher Education. In IACSIT (Ed.), 2nd International Conference on Education and Management Technology (pp. 357-363). Press Singapore. 
Correa, C. (2017). Itinerarios categoriales en la construcción de marcos teóricos. Octaedro.

Downes, S. (2005). E-learning 2.0. https://elearnmag.acm.org/featured.cfm?aid $=1104968$

Drucker, P. (1959). Landmarks of Tomorrow. Harper \& Brothers.

Drucker, P. (1994). The Age of Social Transformation. The Atlantic Monthly, 274(5), 53-80.

Engel, A., Coll, C., Membrive, A., \& Doller, J. (2018). Information and communication technologies and students' out-ofschool learning experiences. Digital Education Review, 33, 130-149. https://doi.org/10.1344/der.2018.33.130-149

Escudero, J., Delfín, L., \& Gutiérrez, L. (2008). El estudio de caso como estrategia de investigación en las ciencias sociales. Ciencias Administrativas, 1, 7-10.

Freire, J. (2010). Educación expandida y nuevas instituciones: ¿es posible la transformación? En R. Díaz y J. Freire (Eds.), Educación expandida (pp. 67-80). Zemos98.

García, L. (2019). Necesidad de una educación digital en un mundo digital. Revista Iberoamericana de Educación a Distancia, $22(2), 9-22$

Gil Antón, M. (2014). Los jóvenes académicos en el siglo XXI: de la oportunidad inesperada al retorno del privilegio. En M. Mora Salas \& O. de Oliveira (Coords.), Desafíos y paradojas. Los jóvenes frente a las desigualdades sociales, (pp. 313-361). El Colegio de México.

Halpern, D. (2003). Thought and Knowledge: An Introduction to Critical Thinking. Lawrence Erlbaum Associates.

Illich, I. (2012). La sociedad desescolarizada. Ediciones Godot.

Jara, N. P., \& Prieto, C. (2018). Impacto de las diferencias entre nativos e inmigrantes digitales en la enseñanza en las ciencias de la salud: revisión sistemática. Revista Cubana de Información en Ciencias de la Salud, 29(1), 92-105.

Krathwohl, D. (2014). Revision of Bloom's Taxonomy. Theory into Practice, 41(4), 212-218.

Linne, J. (2014). Dos generaciones de nativos digitales. Intercom: Revista Brasileira de Ciências da Comunicação, 37(2), 203221. https://dx.doi.org/10.1590/1809-584420149
Litto, F. (2012). Vision about Distance Education. En M. Moreno Castañeda (Coord.), Veinte visiones de la Educación a Distancia (pp. 69-79). UDG Virtual.

Marton, F. (1992). Phenomenography and "the art of teaching all things to all men". International Journal of Qualitative Studies in Education, 5(3), 253-267.

Moravec, J. (2011). Desde la sociedad 1.0 a la sociedad 3.0. En C. Cobo \& J. Moravec (Eds.), Aprendizaje Invisible. Hacia una nueva ecología de la educación (pp. 47-73). Ediciones de la Universidad de Barcelona.

OCDE (2010). Recognition of Non-Formal and Informal Learning: Country Practices. https://www.oecd.org/education/skillsbeyond-school/44600408.pdf

OCDE (2015). México: políticas prioritarias para fomentar las habilidades y el conocimiento de los mexicanos para la productividad y la innovación. https://www.oecd.org/mexico/ mexico-politicas-prioritarias-para-fomentar-las-habilidades-y-conocimientos-de-los-Mexicanos.pdf

OCDE (2020). Mexico Policy Brief: Education and Skills. http:// www.oecd.org/education/Policy-Brief-Mexico-Educationand-Skills-EN.pdf

Piaget, J. (1970). Piaget's Theory. En P. H. Mussen (Ed.). Carmichael's Manual of Child Psychology (Vol. 1). Wiley.

Potter, W. (2010). The State of Media Literacy. Journal of Broadcasting \& Electronic Media, 54(4), 675-696. http://doi.org/10.1080 /08838151.2011.521462

Pozuelo, J. (2014). ¿Y si enseñamos de otra manera? Competencias digitales para el cambio metodológico. Caracciolos, 2(1), $53-75$.

Prata, D., Barbato, S., \& González, M.F. (2020). Ambientes virtuales de aprendizaje y producción de identidad en la formación inicial docente. Digital Education Review, 38, 23-41. https:// doi.org/10.1344/der.2020.38.23-41

Prensky, M. (2001). Digital natives, digital immigrants. On The Horizon, 9(5), 1-6.

Rodriguez-Jimenez, T., \& Vicario, C. M. (2018). Nuevos roles de trabajo y nuevas habilidades en las empresas tecnológicas, su influencia en la educación tecnológica ante la era de las comunidades en Guadalajara, Jalisco. En A. J. Arguellez Cruz, 
C. M. Vicario Solórzano \& P. Gómez Miranda (Comps.), Política y gestión de Tecnología Educativa en México (pp. 41-47). IPN.

Scolari, C. A. (2016). Alfabetismo transmedia: estrategias de aprendizaje informal y competencias mediáticas en la nueva ecología de la comunicación = Transmedia literacy: informal learning strategies and media skills in the new ecology of communication. Telos. (193), 13-23.

Secretaría de Educación Pública (SEP). (30 de octubre de 2000). ACUERDO número 286 por el que se establecen los lineamientos que determinan las normas y criterios generales, a que se ajustarán la revalidación y equivalencia de estudios, así como los procedimientos por medio de los cuales se acreditarán conocimientos correspondientes a niveles educativos o grados escolares adquiridos en forma autodidacta, a través de la experiencia laboral o con base en el régimen de certificación referido a la formación para el trabajo. Diario oficial de la federación, DOF 30-10-2000. https://www.sep. gob.mx/work/models/sep1/Resource/12234/11/images/ Nuevo acuerdo286.pdf

Siemens, G. (2005). Connectivism: A learning theory for the digital age. International Journal of Instructional Technology and Distance Learning, 2(1), 3-10.

UNESCO (2011). Alfabetización mediatica e informacional. Curriculum para profesores. http://unesdoc.unesco.org/ images/0021/002160/216099S.pdf

UNESCO (2015). La Educación Para Todos, 2000-2015: logrosydesafíos. https://unesdoc.unesco.org/ark:/48223/pf0000232435

UNESCO (2017). Informe de seguimiento de la educación en el mundo, 2016. La educación al servicio de los pueblos y el planeta: creación de futuros sostenibles para todos. http://unesdoc.unesco.org/images/0024/002485/248526S.pdf

Uribe, A. (2017). Protoideas educativas de la educación expandida. Revista Virtual Universidad Católica del Norte, 51, 292-310. Vygotsky, L. (1978). Mind in society. Harvard University Press. 